\title{
¿Cuál es la prevalencia de obesidad metabólicamente saludable en población chilena?
}

\author{
What is the prevalence of metabolically healthy obesity in Chile?
}

\begin{abstract}
RESUMEN
La obesidad es un importante factor de riesgo cardiovascular. No obstante, no todas las personas obesas tienen un perfil metabólico alterado ni todas las personas normo-peso poseen un perfil metabólico normal. Objetivo: determinar la prevalencia de diferentes fenotipos metabólicos asociados al estado nutricional en Chile. Métodos: se incluyeron 1.733 participantes de la Encuesta Nacional de Salud 20092010. El estado nutricional (obesidad o normo-peso) fue determinado a través del IMC mientras que la condición metabólica (saludable o no) en base a cuatro parámetros: glicemia, presión arterial, colesterol HDL y triglicéridos. Con estos parámetros de determinaron 4 fenotipos, entre ellos, MUNO: metabólicamente no saludable no obeso y MHO: obeso metabólicamente saludable. Resultados: La prevalencia de $\mathrm{MHO}$ fue de 3,3\% mientras que un $17,4 \%$ presentaba MUNO. Adicionalmente, la prevalencia de $\mathrm{MHO}$ disminuyó en la medida que aumentó la edad y la mayor proporción de individuos metabólicamente saludables se encontraba en el grupo de altos ingresos y con un nivel educacional superior (técnico-universitario). Conclusión: Se evidencia una baja prevalencia de $M H O$, así como también una alta prevalencia de individuos MUNO en la población chilena. Futuras acciones preventivas deberían no sólo considerar el estado nutricional sino también la condición metabólica de la población.

Palabras claves: Enfermedades cardiovasculares, Obesidad, Obesidad metabólicamente saludable, Perfil metabólico.
\end{abstract}

\footnotetext{
ABSTRACT

Obesity is an important cardiovascular risk factor. However, not all obese individuals have an unhealthy metabolic profile and vice versa. Therefore, the aim of this study was to determine the prevalence of different metabolic phenotypes by nutritional status in Chile. Methods: 1,733 individuals from the National Health Survey 2009-10 were included in this study. Nutritional status (obesity or normal-weight) was determined by BMI whereas metabolic profile was determined through four parameters: Glycaemia, blood pressure, HDL cholesterol and triglycerides. Four metabolic phenotypes were derived, among them: MUNO: metabo-
}

Fanny Petermann-Rocha' ${ }^{1}$ Eliana Durán², Ana María Labraña², María Adela Martínez-Sanguinetti ${ }^{3}$, Ana María Leiva ${ }^{4}$, Alex Garrido-Méndez ${ }^{5,6}$, Felipe Poblete-Valderrama ${ }^{7}$, Ximena Díaz ${ }^{8}$, Carlos Salas ${ }^{8}$, Cristian Álvarez ${ }^{10}$, Liliana Cuadra, ${ }^{9}, 11$, Natalia Ulloa ${ }^{11}$, Carlos Celis-Morales ${ }^{12,13}$. en representación del grupo de investigación ELHOC (Epidemiology of Lifestyle and Health Outcomes in Chile).

1. Institute of Health and Wellbeing, University of Glasgow, Glasgow, United Kingdom.

2. Departamento de Nutrición y Dietética y Programa de Magister en Nutrición Humana, Facultad de Farmacia, Universidad de Concepción, Concepción, Chile.

3. Instituto de Farmacia, Facultad de Ciencias, Universidad Austral de Chile, Valdivia, Chile.

4. Instituto de Anatomía, Histología y Patología, Facultad de Medicina, Universidad Austral de Chile, Valdivia, Chile. 5. Departamento de Educación Física, Universidad San Sebastián, Concepción, Chile.

6. Departamento de Ciencias del Deporte y Acondicionamiento Físico, Facultad de Educación, Universidad Católica de la Santísima Concepción, Concepción, Chile.

7. Escuela de Kinesiología, Facultad de Salud,

Universidad Santo Tomás, Sede Valdivia. Chile.

8. Grupo de Investigación Calidad de Vida, Departamento de Educación, Universidad del Biobío, Chillán, Chile.

9. Departamento de Educación Física. Facultad de Educación.

Universidad de Concepción. Concepción, Chile. 10. Núcleo de Investigación en Salud, Actividad Física y Deporte, Universidad de Los Lagos, Osorno, Chile.

11. Centro de Vida Saludable de la Universidad de Concepción, Universidad de Concepción, Concepción, Chile. 12. BHF Glasgow Cardiovascular Research Centre, Institute of Cardiovascular and Medical Science, University of Glasgow, Glasgow, United Kingdom.

13. Centro de Investigación en Fisiología del Ejercicio - CIFE, Universidad Mayor, Santiago, Chile.

Dirigir correspondencia a: Carlos Celis-Morales.

BHF Glasgow Cardiovascular Research Centre, 126 University Avenue, Glasgow University. Glasgow, United Kingdom, G12 8TA Teléfono: +(0)4401413304201 Email: carlos.celis@glasgow.ac.uk

Este trabajo fue recibido el 05 de julio de 2018. Aceptado con modificaciones: 29 de agosto de 2018. Aceptado para ser publicado: 07 de enero de 2019.

lically unhealthy and non-obese and MHO: metabolically healthy obesity. Results: The prevalence of MHO in the 
Chilean population was $3.3 \%$ while the prevalence of MUNO was $17.4 \%$. Moreover, the prevalence of $\mathrm{MHO}$ decreased as age increased and a greater proportion of metabolically healthy individuals were in the highest gross income group and in the technical-university educational level. Conclusion: This study shows a low prevalence of $\mathrm{MHO}$ and a higher prevalence of MUNO in the Chilean population. Future preventive actions should take into account not only the nutritional status, but also the metabolic profile of the population. Keywords: Cardiovascular disease, Metabolically healthy obesity, Metabolic profiling, Obesity.

\section{INTRODUCCIÓN}

La obesidad se define como una acumulación anormal o excesiva de grasa corporal perjudicial para la salud'. En los últimos años ésta ha mostrado una evolución dramáticamente creciente en la gran mayoría de los países a nivel mundial, siendo responsable de un sinnúmero de complicaciones que impactan directamente sobre la calidad y expectativa de vida de las personas que la padecen $^{1,2,3}$. Según cifras de la Organización Mundial de la Salud (OMS), en el año 2016 más de 650 millones de individuos padecían obesidad en todo el mundo, es decir, presentaban un índice de masa corporal (IMC) $\geq 30,0 \mathrm{~kg} /$ $\mathrm{m}^{2(1,4)}$. No obstante, $y$ a pesar de estas desalentadoras cifras de prevalencia, estudios internacionales han estimado que un porcentaje de individuos obesos podrían poseer un menor riesgo de desarrollar cualquiera de las alteraciones metabólicas relacionadas con la obesidad (como diabetes mellitus tipos 2, hipertensión arterial y/o dislipidemia), al menos durante un período desconocido de tiempo ${ }^{5,6,7}$. A este subgrupo de individuos se les ha identificado como Obesos Metabólicamente Saludables (Metabolically-Healthy Obesity, MHO por su sigla en inglés).

Los individuos con $\mathrm{MHO}$ muestran un perfil metabólico normal caracterizado por niveles normales en el perfil lipídico, insulina y presión arterial, junto con un bajo nivel de respuestas inflamatorias sistémicas ${ }^{6,8}$. En este contexto, estos individuos a pesar de presentar obesidad no exhiben otras alteraciones metabólicas directamente relacionadas con esta patología, muy probablemente porque aquellos individuos $\mathrm{MHO}$ poseen una menor cantidad de grasa visceral en comparación a individuos obesos metabólicamente no saludables (Metabollicaly unhealthy obesity, MUO), lo cual se relaciona directamente con resistencia a la insulina ${ }^{6,8}$. Adicionalmente, estudios previos también han identificado a un subgrupo de individuos normo-peso con parámetros metabólicos anormales (conocidos como normo-peso o no-obesos metabólicamente no saludables, Metabollicaly unhealthy non-obese: MUNO) ${ }^{9}$. Es así como un rango de fenotipos metabólicamente saludable y no saludables, obesos, y no obesos, existen y han sido reconocidos desde $1980^{6}$.

La prevalencia de $\mathrm{MHO}$ varía mucho entre poblaciones, alcanzando cifras que van desde el 6 al $75 \%$ de las poblaciones estudiadas $s^{5,6,7,8,9,10}$. Esta gran diferencia entre prevalencia se debe, principalmente, a los diferentes criterios metabólicos utilizados para definirla, sin existir hasta ahora una consistente definición ${ }^{6}$. En Chile, y a pesar de que según la última Encuesta Nacional de Salud (ENS) 20162017 un 33,4 \% de la población chilena padece obesidad según IMC ${ }^{11}$, la presencia de $\mathrm{MHO}$ o MUNO no ha sido investigada ni se ha estimado la prevalencia de individuos que podrían presentarla. Por esta razón, el objetivo de este estudio fue determinar la prevalencia de diferentes fenotipos metabólicos asociados con el estado nutricional, entre ellos obesos metabólicamente saludable, en población chilena.

\section{MATERIALES Y METODOS \\ Diseño del estudio}

La muestra seleccionada comprendió a los participantes de la Encuesta Nacional de Salud desarrollada entre octubre del año 2009 y septiembre del año 2010 (ENS 2009-2010) ${ }^{12}$. La ENS 2009-2010 correspondió a un estudio de prevalencia realizado en hogares en una muestra nacional, probabilística, estratificada y multietápica de 5.412 personas mayores de 15 años con representatividad nacional, regional, y área urbana/ rural. Se incluyeron un total de 1.733 participantes mayores de 18 y menores de 65 años con información disponible en relación con su IMC y variables metabólicas (glicemia basal, triglicéridos, colesterol HDL y presión arterial). No se incluyeron participantes con estado nutricional de bajo peso.

El protocolo de la ENS 2009-2010 fue aprobado por el Comité de Ética de Investigación de la Escuela de Medicina de la Pontificia Universidad Católica de Chile. Todos los participantes firmaron un consentimiento informado ${ }^{12}$.

\section{Mediciones antropométricas y metabólicas}

El peso corporal fue medido a través de una balanza digital (Tanita HD-313®) y la talla a través de un tallímetro con cartabón incorporado, sin zapatos y utilizando ropa ligera. Con estas mediciones se obtuvo el estado nutricional el cual fue clasificado en base a los puntos de corte del IMC según los criterios de la $\mathrm{OMS}^{4}$, mientras que la obesidad central fue definida como un perímetro de cintura $\geq 83 \mathrm{~cm}$ $y \geq 88 \mathrm{~cm}$ en mujeres y hombres, respectivamente ${ }^{12}$.

Las muestras sanguíneas fueron obtenidas por punción venosa luego un periodo de entre 10 y 12 horas de ayuno, por una enfermera entrenada siguiendo protocolos estandarizados a nivel nacional, tal como ha sido previamente descrito en la ENS ${ }^{12}$. La presión arterial fue medida por personal entrenado y derivada tras tres mediciones luego 
de 15 minutos de reposo a través de un tensiómetro digital automático (Omron HEM 742®).

\section{Fenotipos metabólicos}

El estado nutricional (normal u obesidad) fue clasificado en base al IMC, como ha sido descrito anteriormente. A su vez, cada individuo fue clasificado en base a su condición metabólica (saludable o no saludable). Individuos con sobrepeso metabólicamente saludables o no saludables metabólicamente fueron incluidos para estimar la prevalencia total de $\mathrm{MHO}$ en la población, a pesar de que las características sociodemográficas de estos grupos no sean presentadas (Figuras 1 y 2).

Individuos metabólicamente saludables cumplieron con los siguientes criterios: glicemia en ayuno $<100 \mathrm{mg} / \mathrm{dl}$, presión arterial sistólica (PAS) $<135 \mathrm{~mm} \mathrm{Hg}$ y/o una presión arterial diastólica (PAD) $<85 \mathrm{~mm} \mathrm{Hg}$, colesterol HDL $>40$ $\mathrm{mg} / \mathrm{dl}$ en hombres y $>50 \mathrm{mg} / \mathrm{dl}$ en mujeres, y triglicéridos $<150 \mathrm{mg} / \mathrm{dl}$. Esta definición fue basada en la clasificación de síndrome metabólico sugerida por la IDF-ATP ${ }^{12,13}$. A partir de estos criterios (estado nutricional y condición metabólica), se obtuvo cuatros fenotipos metabólicos: MHNO: metabólicamente saludable no obeso; MUNO: metabólicamente no saludable no obeso; MHO: obeso metabólicamente saludable; MUO: obeso metabólicamente no saludable.

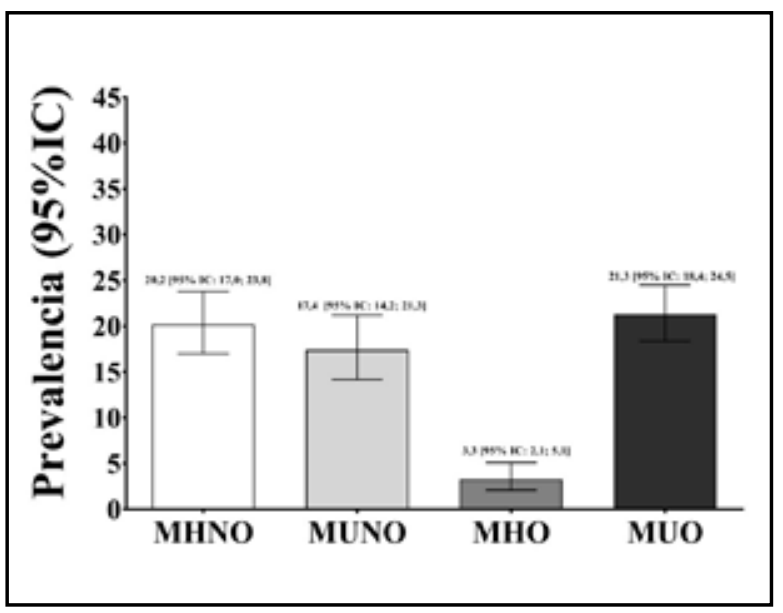

Figura 1. Prevalencia de los distintos fenotipos metabólicos en población chilena. Prevalencia del estado nutricional (normal u obesidad) en base a condición metabólica (saludable o no saludable). Individuos metabólicamente saludables presentaban todos los siguientes parámetros normales: presión arterial, glicemia basal, colesterol-HDL y triglicéridos. Los datos son presentados como proporción con sus respectivos $95 \%$ de intervalo de confianza ( $95 \% \mathrm{IC})$. MHNO: metabólicamente saludable no obeso; MUNO: metabólicamente no saludable no obeso; MHO: obeso metabólicamente saludable; MUO: obeso metabólicamente no saludable.

\section{Variables sociodemográficas y de estilo de vida}

Las variables sociodemográficas como edad, sexo, zona geográfica de residencia (urbano/rural), nivel de escolaridad (básica $<8$ años, media: 8 a 12 años, y técnica-universitaria $>12$ años) e ingreso económico (bajo <250.000; medio 250.000 a 450.000 ; alto $>450.000$ pesos chilenos) fueron recolectadas mediante el uso de cuestionarios validados en población nacional. Los datos asociados con los estilos de vida, como el tabaquismo, consumo de frutas, y verduras, horas de sueño $(<7$ horas, $7-9$ horas y $>9$ horas) y autoreporte de salud y bienestar (malo, regular o bueno) se obtuvieron mediante la aplicación de cuestionarios validados en la ENS ${ }^{12}$. El consumo de sal se determinó mediante análisis de excreción de sodio en la orina, a través de la fórmula de Tanaka $2002^{14}$. Los niveles de actividad física fueron determinados con el cuestionario "Global Physical Activity Questionnaire"15. Los niveles de sedentarismo fueron determinados mediante el auto-reporte de tiempo

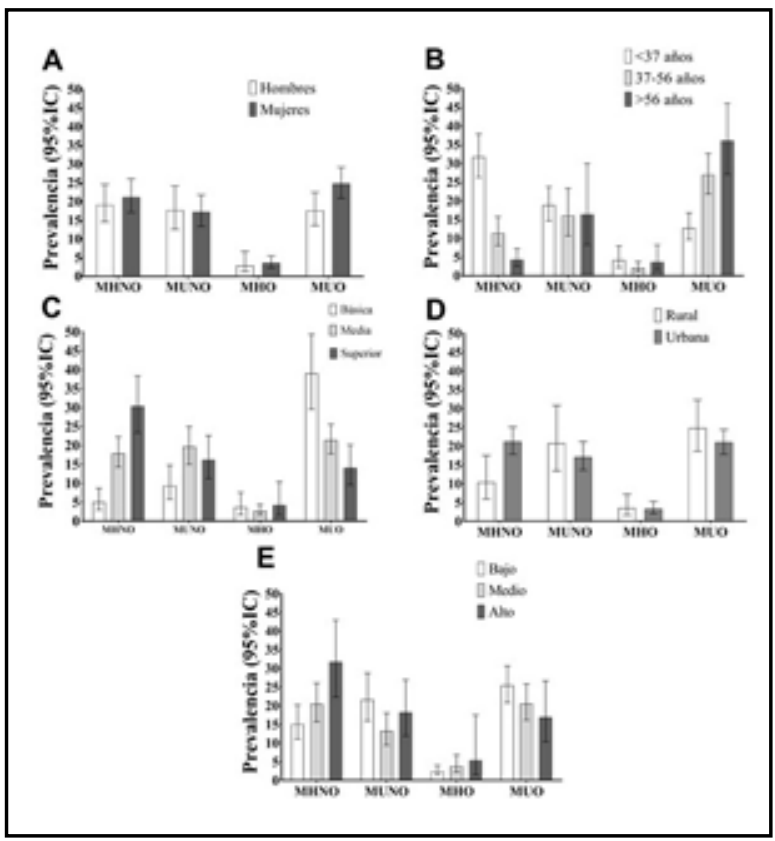

Figura 2. Prevalencia de los distintos fenotipos metabólicos en Chile según distintos factores sociodemográficos. Individuos metabólicamente saludables (con estado nutricional normal o de obesidad) presentaban todos los siguientes parámetros normales: presión arterial, glicemia basal, colesterol total, colesterol-HDL y triglicéridos. La figura A muestra la prevalencia según sexo; la figura B la prevalencia según escolaridad; figura C la prevalencia según nivel educacional; figura $\mathrm{D}$ la prevalencia según región de origen; y figura E la prevalencia según nivel de ingresos. Los datos son presentados como proporción con sus respectivos $95 \%$ de intervalo de confianza $(95 \% \mathrm{IC})$. MHNO: metabólicamente saludable no obeso; MUNO: metabólicamente no saludable no obeso; MHO: obeso metabólicamente saludable; MUO: obeso metabólicamente no saludable. 
destinado a actividades que involucren estar sentado o reclinado durante el tiempo libre o de trabajo, a través del mismo cuestionario. Se consideró como punto de corte para inactividad física un gasto energético menor a $<600$ METs/minutos/semana, según las recomendaciones de la OMS y especificaciones en la guía de análisis de GPAQ ${ }^{15,16}$.

\section{Análisis estadístico}

Los datos de caracterización de la población estudiada son presentados como promedio con sus respectivos $95 \%$ de intervalo de confianza $(95 \%$ IC) para variables continuas, y como porcentaje con sus respectivos $95 \%$ IC para variables categóricas (Tabla 1). Para todos los análisis se utilizó el programa STATA MP v14 y el comando de "svyset" para muestras complejas (https://www.stata.com/ manuals ${ }^{13} /$ svy.pdf). Se tomó en consideración los factores de expansión sugeridos por la ENS 2009-2010, ya que todos los resultados fueron estimados utilizando muestras expandidas según la ENS ${ }^{12}$.

\section{RESULTADOS}

Las características generales de los participantes, según estado nutricional y condición metabólica (MHNO, MUNO, $\mathrm{MHO}$ y MUO), se presentan en la tabla 1. En comparación al grupo de referencia (MHNO), los individuos MUNO, $\mathrm{MHO}$ y MUO presentaban una mayor edad y un mayor porcentaje vivía en zonas rurales. A nivel educacional, un mayor porcentaje de individuos MHNO poseía un nivel educacional más alto, en comparación a los individuos con alteraciones metabólicas y/u obesidad. Económicamente, individuos, metabólicamente saludables (MHNO o MHO), presentaron un mayor porcentaje de personas con un ingreso medio-alto en comparación con los grupos metabólicamente no saludables. Antropométricamente, el perímetro de cintura aumentó en la medida que aumentaron las variables metabólicas de riesgo y el IMC. Finalmente, y en relación con el estilo de vida, los individuos MHNO presentaban una menor prevalencia de inactividad física en comparación a sus pares MUNO. Adicionalmente, individuos MHO consumían menos alcohol que MUO (39,5 g/día versus 69,3 g/día) y un mayor porcentaje presentó un mejor reporte de salud y bienestar.

En relación con la prevalencia de estos 4 fenotipos metabólicos, se destaca que un 3,3\% [95\% IC: 2,1; 5,1] de la población estudiada presentaba $\mathrm{MHO}$ (obesidad sin alteraciones metabólicas) y un 17,4\% [95\% IC: 14,2; 21,3] MUNO (estado nutricional normal con variables metabólicas alteradas) (Figura 1).

Al evaluar la prevalencia de estos 4 fenotipos según distintas variables sociodemográficas (Figura 2), y en relación al total de la muestra, se evidencia que, en comparación a los hombres, las mujeres presentaron una mayor prevalencia de $\mathrm{MHNO}, \mathrm{MHO}$ y $\mathrm{MUO}(21,2 \%$, $3,6 \%$ y $24,8 \%$, respectivamente) (Figura $2 \mathrm{~A}$ ), la prevalencia de $\mathrm{MHO}$ disminuyó en la medida que aumentó la edad $(4,2 \%<37$ años versus $3,7 \%>56$ años, Figura $2 \mathrm{~B})$ y la mayor proporción de individuos metabólicamente saludables (ya sea MHNO o MHO) se encontraba en el grupo de altos ingresos y con un nivel educacional medio o técnico-universitario (Figura $2 \mathrm{C}$ y $2 \mathrm{E}$ ). Finalmente, al evaluar la proporción de individuos acorde a su zona de residencia (Figura 2D), se destaca que una mayor proporción de individuos metabólicamente no saludable vive en zonas rurales, en comparación con su referente saludable $(20,8 \%$ MUNO versus $10,3 \%$ MHNO y $24,8 \%$ MUO versus 3,5\% MHO).

\section{DISCUSIÓN}

El aumento de la prevalencia de obesidad es un desafío y una preocupación mundial ${ }^{2}$. Mientras las acciones dirigidas a frenar factores de riesgo vascular modificables, como el tabaquismo, la hipertensión arterial o la dislipidemia, han avanzado con mayor o menor grado de éxito, la obesidad ha avanzado de una manera progresiva, con especial intensidad en los grados de obesidad más severos ${ }^{3}$. Sin embargo, los resultados presentados en este estudio señalan que no todas las personas obesas presentan alteraciones metabólicas, así como también dejan en manifiesto que no todas las personas con un estado nutricional normal estarían protegidas de riesgo metabólico. En otras palabras, poseer un estado nutricional normal no siempre es sinónimo de buena salud metabólica. Sin duda, estas controversias identificadas entre estado nutricional y estado metabólico se deben, fundamentalmente, a la actual determinación del estado nutricional a través del parámetro IMC. Este indicador, a pesar de ser una herramienta fácil de usar y de bajo costo, podría presentar limitaciones al momento de identificar sujetos con un IMC normal, pero que podrían presentar un alto nivel de adiposidad visceral, lo cual probablemente explicaría la presencia de un perfil metabólico alterado ${ }^{17}$.

En relación con la prevalencia, en este estudio se determinó que un 3,3\% de los obesos presentaba una $\mathrm{MHO}$ al utilizar los puntos de corte de la IDF-ATP ${ }^{13}$. La literatura ha evidenciado una gran oscilación relacionada con esta prevalencia (entre un 6 a un $75 \%)^{5-10}$, principalmente por los diferentes criterios, puntos de corte utilizados y la cantidad de parámetros considerados para su clasificación. Un claro ejemplo de esto se evidencia al comparar nuestros resultados con los obtenidos por Meigs y Cols. en el año $2006^{18}$, quienes, a pesar de utilizar parámetros y puntos de corte similares para variables metabólicas, clasificaron a individuos como obeso metabólicamente saludable cuando presentaban alteración en menos de 3 de las 5 variables que ellos estudiaron, es decir, ausencia de síndrome metabólico. Con estos puntos de corte Meigs y Cols. identificaron una prevalencia de un $7 \%$ de MUNO y de un $37 \%$ de $\mathrm{MHO}^{18}$. Situación similar fue observada por Widman y Cols., quienes identificaron una prevalencia de un $23,5 \%$ de $M U N O$ y de un $31,7 \%$ de $\mathrm{MHO}^{19}$. En este contexto, el diagnóstico de MHO con sólo 3 de 5 de las variables estudiadas, o incluso menos parámetros 
Tabla 1. Características de la población según condición metabólica.

\begin{tabular}{|c|c|c|c|c|}
\hline $\begin{array}{l}\text { Sociodemográficas } \\
\mathrm{n}\end{array}$ & $\begin{array}{c}\text { MHNO } \\
281\end{array}$ & $\begin{array}{c}\text { MUNO } \\
252\end{array}$ & $\begin{array}{c}\text { MHO } \\
63\end{array}$ & $\begin{array}{l}\text { MUO } \\
443\end{array}$ \\
\hline Mujeres (\%) & $53,9(44,5 ; 63,0)$ & $50,6(39,2 ; 62,0)$ & $56,3(33,0 ; 77,2)$ & $59,7(51,8 ; 67,2)$ \\
\hline Edad (años) & $28,6(25,6 ; 31,6)$ & $38,9(33,7 ; 44,0)$ & $31,9(16,8 ; 47,0)$ & $39,2(35,8 ; 42,6)$ \\
\hline \multicolumn{5}{|l|}{ Zona de residencia (\%) } \\
\hline Urbana & $95,1(91,5 ; 97,3)$ & $88,6(81,9 ; 93,1)$ & $89,9(78,7 ; 95,6)$ & $88,9(85,4 ; 91,7)$ \\
\hline \multicolumn{5}{|l|}{ Nivel educacional (\%) } \\
\hline Básica & $2,9(1,7 ; 5,0)$ & $6,2(3,7 ; 10,0)$ & $12,8(5,6 ; 25,6)$ & $21,1(15,6 ; 27,9)$ \\
\hline Media & $52,1(42,7 ; 61,2)$ & $66,1(55,7 ; 75,2)$ & $48,7(28,1 ; 69,7)$ & $59,1(51,2 ; 66,5)$ \\
\hline Técnico Universitaria & $45,0(35,9 ; 54,5)$ & $27,7(19,4 ; 37,9)$ & $38,5(19,4 ; 37,9)$ & $19,8(13,9 ; 27,4)$ \\
\hline \multicolumn{5}{|l|}{ Nivel de ingreso (\%) } \\
\hline Bajo & $33,7(25,3: 43,3)$ & $54,3(43,2: 65,0)$ & $31,5(17,4: 50,1)$ & $51,4(43,6: 59,0)$ \\
\hline Medio & $39,0(30,4: 48,3)$ & $28,2(20,1: 37,9)$ & $42,2(23,3: 63,6)$ & $35,5(28,5: 43,1)$ \\
\hline Alto & $27,3(19,1: 37,4)$ & $17,5(11,3: 26,3)$ & $26,3(8,4: 58,3)$ & $13,1(8,1: 20,8)$ \\
\hline \multicolumn{5}{|l|}{ Antropométricas } \\
\hline Peso $(\mathrm{kg})$ & $61,2(57,7 ; 64,7)$ & $66,5(60,8 ; 72,2)$ & $85,1(79,8 ; 90,3)$ & $92,1(84,6 ; 99,7)$ \\
\hline Talla $(\mathrm{cm})$ & $1,66(1,63 ; 1,70)$ & $1,67(1,61 ; 1,74)$ & $1,62(1,55 ; 1,69)$ & $1,63(1,60 ; 1,67)$ \\
\hline $\mathrm{IMC}\left(\mathrm{kg} / \mathrm{m}^{2}\right)$ & $22,0(21,2 ; 22,8)$ & $23,6(23,1 ; 24,1)$ & $32,3(30,8 ; 33,8)$ & $34,4(32,2 ; 36,6)$ \\
\hline Perímetro de cintura $(\mathrm{cm})$ & $76,6(74,1 ; 79,1)$ & $83,6(79,7 ; 87,5)$ & $96,7(91,2 ; 102,3)$ & $105,8(100 ; 111,6)$ \\
\hline Obesidad central (\%) & $4,2(1,3 ; 12,5)$ & $24,3(13,9 ; 38,9)$ & $63,7(42,6 ; 80,6)$ & $71,0(63,9 ; 77,3)$ \\
\hline \multicolumn{5}{|l|}{ Estilos de vida } \\
\hline \multicolumn{5}{|l|}{ Actividad física total } \\
\hline (MET/hora/semana) & $131,0(81,9 ; 180,2)$ & $93,8(54,7 ; 132,9)$ & $81,7(30,8 ; 132,6)$ & $123,1(82,8 ; 163,3)$ \\
\hline Prevalencia Inactividad física (\%) & $12,4(7,8 ; 19,1)$ & $13,2(8,8 ; 19,4)$ & $14,0(6,6 ; 27,1)$ & $20,8(15,2 ; 27,7)$ \\
\hline Tiempo sedente (hora/día) & $3,9(3,0 ; 4,9)$ & $2,8(2,0 ; 3,6)$ & $2,0(1,6 ; 2,3)$ & $4,2(3,2 ; 5,2)$ \\
\hline \multicolumn{5}{|l|}{ Consumo de frutas y vegetales } \\
\hline (g/día) & $232,4(172,3 ; 292,4)$ & $226,9(148,9 ; 305,0)$ & $264,5(204,0 ; 325,0)$ & $223,3(173,6 ; 273,0)$ \\
\hline Consumo de alcohol (g/día) & $75,8(49,7 ; 101,8)$ & $43,2(21,3 ; 65,2)$ & $39,5(29,2 ; 49,9)$ & $69,3(36,2 ; 102,4)$ \\
\hline Consumo de sal (g/día) & $8,1(7,3 ; 8,9)$ & $10,3(8,7 ; 12,0)$ & $11,4(8,7 ; 14,0)$ & $10,7(9,9 ; 11,5)$ \\
\hline \multicolumn{5}{|l|}{ Tabaquismo (\%) } \\
\hline Nunca & $29,5(22,4 ; 37,9)$ & $28,8(19,3 ; 40,4)$ & $35,6(19,0 ; 56,7)$ & $31,7(25,1 ; 39,1)$ \\
\hline Ex-fumador & $21,6(14,8 ; 30,3)$ & $16,5(9,6 ; 26,9)$ & $12,2(5,2 ; 26,0)$ & $31,4(24,8 ; 38,8)$ \\
\hline Fumador & $48,9(39,6 ; 5,2)$ & $54,7(43,1 ; 65,9)$ & $52,2(31,1 ; 72,5)$ & $36,9(29,8 ; 44,6)$ \\
\hline \multicolumn{5}{|l|}{$\begin{array}{l}\text { Auto-reporte de salud y } \\
\text { bienestar }(\%)\end{array}$} \\
\hline Malo & $2,8(1,1 ; 7,1)$ & $7,1(2,3 ; 19,7)$ & $0,4(0,1 ; 1,9)$ & $2,7(1,2 ; 5,5)$ \\
\hline Regular & $23,8(16,4 ; 33,2)$ & $26,9(18,2 ; 37,9)$ & $18,5(8,4 ; 35,8)$ & $38,5(31,3 ; 46,3)$ \\
\hline Bueno & $73,4(64,0 ; 81,1)$ & $66,0(54,1 ; 76,2)$ & $81,1(63,8 ; 91,3)$ & $58,8(51,1 ; 66,1)$ \\
\hline \multicolumn{5}{|l|}{ Horas de sueño (\%) } \\
\hline 7-9 horas & $47,6(38,6 ; 56,8)$ & $47,1(35,8,58,6)$ & $43,3(24,7 ; 63,9)$ & $46,2(38,8 ; 53,8)$ \\
\hline$<7$ horas & $31,9(23,7 ; 41,4)$ & $31,1(21,7 ; 42,3)$ & $38,1(17,9 ; 63,5)$ & $41,5(34,0 ; 49,4)$ \\
\hline$>9$ horas & $20,5(13,5 ; 30,0)$ & $21,8(13,5 ; 33,3)$ & $18,6(7,3 ; 40,0)$ & $12,3(8,4 ; 17,7)$ \\
\hline $\begin{array}{l}\text { Datos presentados como promedi } \\
\text { y como porcentaje con sus respec } \\
\text { MUNO: metabólicamente no salud } \\
\text { no saludable. Individuos obesos me } \\
\text { arterial normal, glicemia normal, } \\
\text { saludables o no saludables metab }\end{array}$ & $\begin{array}{l}\text { ble no obeso; } \mathrm{MHO} \\
\text { abólicamente salud } \\
\text { lesterol-HDL y trig } \\
\text { licamente no fueror }\end{array}$ & $\begin{array}{l}\text { écidos normales. Parti } \\
\text { cluidos en esta tabla }\end{array}$ & $\begin{array}{l}\text { saludable; MUO: obe } \\
\text { suientes criterios: IMC } \geq \\
\text { icipantes con sobrepes } \\
(n=694) \text {. }\end{array}$ & $\begin{array}{l}\text { variables continuas } \\
\text { saludable no obeso } \\
\text { eso metabólicament } \\
\geq 30,0 \mathrm{~kg} / \mathrm{m} 2 \text {, presiór } \\
\text { so metabólicament }\end{array}$ \\
\hline
\end{tabular}


metabólicos, podría explicar la menor prevalencia identificada de $\mathrm{MHO}$ y mayor prevalencia de MUNO en población chilena, ya que en nuestro estudio, individuos metabólicamente saludables debían presentar todos los parámetros metabólicos estudiados normales y no solamente 2 ó 3 de ellos. Situación similar al reciente estudio publicado por Eckel y Cols., en la corte del estudio de seguimiento a enfermeras (Nurses' Health Study), quienes definieron MHO como la ausencia total de cualquier alteración metabólica (ya sea hipertensión arterial, diabetes mellitus tipo 2 y/o dislipidemia), evidenciando una prevalencia de 9,6\% de MUNO y de un $6,3 \%$ de $\mathrm{MHO}^{20}$, cifras más cercanas a las identificadas en este estudio.

Por otro lado, y en relación con la prevalencia según variables sociodemográficas, estudios previos también han mostrado que las mujeres presentan una mayor prevalencia de $\mathrm{MHO}^{21,22}$ y que la proporción de sujetos metabólicamente sanos desciende con la edad. El grupo español EPINUT coincide en estas conclusiones, encontrando un descenso particularmente significativo a partir de los 45 años $^{21}$.

Datos longitudinales muestran claramente que no todos los individuos $\mathrm{MHO}$ permanecen metabólicamente saludables en el tiempo, convirtiéndose en MUO en un período de 5 a10 años. En esta línea, Eckel y Cols., en el estudio de seguimiento de las enfermeras, demostraron que aquellas mujeres que mantuvieron su fenotipo de $\mathrm{MHO}$ durante los 30 años de seguimiento, presentaban un mayor riesgo de enfermedad cardiovascular, en comparación con las mujeres $\mathrm{MHNO}$; sin embargo, este riesgo fue menor que el de aquellas mujeres que inicialmente se encontraban metabólicamente sanas y que durante el seguimiento se convirtieron a un fenotipo metabólico no saludable ${ }^{20}$. Se ha sugerido que el envejecimiento y el aumento de peso corporal podrían estar asociados a esta conversión. Inversamente, un mayor tiempo de actividad física y pérdida de peso corporal estarían asociados con una mayor probabilidad de preservar el fenotipo de $\mathrm{MHO}$, acciones que, al mismo tiempo, son parte de las directrices actuales del tratamiento de la obesidad ${ }^{22}$.

Entre las limitaciones de este estudio se encuentra el número total de participantes en los cuales se pudo determinar fenotipos metabólicos, ya que a pesar que la ENS 2009-10 fue estudiada en 5.412 personas, solamente 1.733 cumplían con los criterios de edad y poseían información metabólica acorde a las variables estudiadas, lo que pudo haber limitado el poder de los resultados. También cabe mencionar que hay otras variables metabólicas de interés que no fueron medidas y que si bien, los cuatro marcadores metabólicos utilizados podrían estar en estado de normalidad, no podemos destacar que otros parámetros no lo estuvieran, tales como la concentración de insulina, y proteína C-reactiva. Por otro lado, y a pesar de ser la ENS 2009-2010 una muestra representativa de la realidad chilena, la información fue recolectada hace más de 9 años $y$, considerando el acelerado aumento en la prevalencia de obesidad asociado al cambio en los estilos de vida, esta información podría cambiar con la actualización de los nuevos datos de la ENS 20016-2017.

\section{CONCLUSIÓN}

Los resultados de este estudio entregan evidencia de la prevalencia de distintos fenotipos metabólicos según estado nutricional en población chilena, manifestado una baja prevalencia de $\mathrm{MHO}$, así como también una mayor prevalencia de individuos no obesos, pero metabólicamente no saludables. Considerando las actuales cifras de obesidad en Chile, y las proyecciones futuras, potenciar la adopción de estilos de vida que fomenten conductas protectoras y permitan disminuir las variables de riesgo, asociadas a alteraciones metabólicas, podría jugar un rol fundamental, no solamente para mantener por un mayor tiempo a individuos $\mathrm{MHO}$, sino también para disminuir las complicaciones en aquellos individuos que a simple vista no presentan alteraciones, pero que incluso podrían encontrarse en mayor riesgo de sufrir un evento cardiovascular que aquellos $\mathrm{MHO}$.

Agradecimientos. Se agradece de manera especial a todos los participantes de la ENS 2009-10, al equipo profesional de la Escuela de Salud Pública, de la Facultad de Medicina de la Pontíficia Universidad Católica de Chile, quienes desarrollaron y aplicaron la Encuesta Nacional de Salud y al Ministerio de Salud del Gobierno de Chile. NU y LC agradecen al Convenio de Desempeño de la Universidad de Concepción y MINEDUC, CD UCO1201. Todos los autores revisaron críticamente el manuscrito y están de acuerdo con su versión final. Ninguno de los autores reporta tener conflictos de intereses.

\section{BIBLIGRAFÍA}

1. WHO. 10 facts on obesity. World Health Organization, 2017. Available: http://www.who.int/features/factfiles/obesity/en/

2. Zobel E., Hansen T., Rossing P., von Scholten B. Global Changes in Food Supply and the Obesity Epidemic. Current obesity reports, 2016; 5(4): 449-455.

3. Álvarez J. R. M. Advances in feed, nutrition and dietetic. SEDCA nutrición 2012. Available: http://www.nutricion. org/publicaciones/publicacion. asp?id=60

4. WHO. Obesity: Preventing and Managing the Global Epidemic. World Health Organization 2000. Available: http://www. who.int/nutrition/publications/obesity/WHO_TRS_894/en/

5. Rey-López J., Rezende L., Pastor-Valero M., Tess B. The prevalence of metabolically healthy obesity: A systematic review and critical evaluation of the definitions used. Obesity reviews, 2014; 15(10): 781-790.

6. Jung $\mathrm{CH}$, Lee WJ, Song KH. Metabolically healthy obesity: A friend or foe? The Korean journal of internal medicine. 2017; 32(4): 611-621.

7. Martínez-Larrad, M., Anchuelo, A., Del Prado, N., Rueda, J., Gabriel, R., \& Serrano-Ríos, M. Profile of individuals who are metabolically healthy obese using different definition criteria. A population-based analysis in the Spanish population. PloS one, 2014; 9(9): e106641.

8. Seo M., Rhee E. Metabolic and cardiovascular implications of a metabolically healthy obesity phenotype. Endocrinology 
and metabolism, 2014; 29(4): 427-434.

9. Eckel N., Meidtner K., Kalle-Uhlmann T., Stefan N., Schulze, M. Metabolically healthy obesity and cardiovascular events: A systematic review and meta-analysis. European journal of preventive cardiology 2016; 23(9): 956-966.

10. Bluher M. The distinction of metabolically 'healthy' from 'unhealthy' obese individuals. Curr Opin Lipidol. 2010; 21(1): 38-43.

11. MINSAL. National Health Survey 2016-2017. Ministerio de Salud, Gobierno de Chile, 2010. Available:http://www.minsal. cl/wp-content/uploads/2017/11/ENS-2016-17_PRIMEROSRESULTADOS.pdf

12. MINSAL. National Health Survey 2009-2010. Ministerio de Salud, Gobierno de Chile, 2010. Available: http://www.minsal. cl/portal/url/item/bcb03d7bc28b64dfe040010165012d23. $p d f$

13. Alberti KGMM, Eckel RH, Grundy SM, et al. Harmonizing the Metabolic Syndrome A Joint Interim Statement of the International Diabetes Federation Task Force on Epidemiology and Prevention; National Heart, Lung, and Blood Institute; American Heart Association; World Heart Federation; International Atherosclerosis Society; and International Association for the Study of Obesity. Circulation 2009; 120(16): 1640-1645.

14. Tanaka T, Okamura T, Miura K, Kadowaki T, Ueshima H, Nakagawa $H$, et al. A simple method to estimate populational 24-h urinary sodium and potassium excretion using a casual urine specimen. Journal of human hypertension. 2002; 16(2): 97-103.

15. WHO. Global Physical Activity Questionnaire: GPAQ version 2.0. World Health Organization, 2009. Available: http://www.who.int/ncds/surveillance/steps/resources/ GPAQ_Analysis_Guide.pdf

16. WHO. Global recommendations on physical activity for health. World Health Organization, 2010. Available: http://www. who.int/dietphysicalactivity/factsheet_recommendations/ en/

17. Bosello O., Donataccio M., Cuzzolaro M. Obesity or obesities? Controversies on the association between body mass index and premature mortality. Eating and Weight Disorders-Studies on Anorexia, Bulimia and Obesity 2016; 21(2): 165-174.

18. Meigs JB, Wilson PW, Fox CS, Vasan RS, Nathan DM, Sullivan $L M$, et al. Body mass index, metabolic syndrome, and risk of type 2 diabetes or cardiovascular disease. The Journal of clinical 19. endocrinology and metabolism. 2006; 91(8): 2906-2912.

19. Wildman RP, Muntner P, Reynolds K, McGinn AP, Rajpathak $S$, Wylie-Rosett J, et al. The obese without cardiometabolic risk factor clustering and the normal weight with cardiometabolic risk factor clustering: prevalence and correlates of 2 phenotypes among the US population (NHANES 1999-2004). Archives of internal medicine. 2008; 168(15): 1617-1624.

20. Eckel N, Li Y, Kuxhaus O, Stefan N, Hu FB, Schulze MB. Transition from metabolic healthy to unhealthy phenotypes and association with cardiovascular disease risk across BMI categories in 90257 women (the Nurses' Health Study): 30 year follow-up from a prospective cohort study. The Lancet Diabetes \& Endocrinology. 2018; 6(9): 714-724.

21. Marrodán-Serrano M., Martínez-Álvarez J., Sánchez-Álvarez M., López-Ejeda N., Alférez García I., Villarino Marín A. Prevalence of metabolically healthy phenotype among adult Spaniards with excess weight. Revista Española de Cardiología 2016: 69(02): 216-217.

22. Donini L., Merola G., Poggiogalle E., Lubrano C., Gnessi L., Mariani S., et al. Disability, Physical Inactivity, and Impaired Health-Related Quality of Life Are Not Different in Metabolically Healthy vs. Unhealthy Obese Subjects. Nutrients 2016; 8(12): 759.

23. Samocha-Bonet D., Dixit V., Kahn C., Leibel L., Lin X., Nieuwdorp M., et al. Metabolically healthy and unhealthy obese-the 2013 Stock Conference report. Obesity reviews, 2014;15(9): 697-708. 